\title{
ERCP in late post-traumatic biliary fistula
}

\author{
G.R.Barclay and J.R. Crampton \\ Department of Medicine, Hope Hospital, (University of Manchester School of Medicine), Eccles Old Road, \\ Salford M6 8HD, UK.
}

\begin{abstract}
Summary: A case is described in which a huge subcapsular bile collection due to biliary fistula presented late after hepatic trauma and in which ERCP indicated the diagnosis.
\end{abstract}

\section{Introduction}

Biliary fistulae are rare sequelae of hepatic trauma the majority of which occur early and settle spontaneously. We report the case of a patient presenting late after the original trauma with a bile collection due to a fistula in whom the site of the fistula was clearly demonstrated by endoscopic retrograde cholangiopancreatography (ERCP). We discuss the value of this approach to the definitive diagnosis and management of this condition.

\section{Case report}

A 35 year old male engineer sustained blunt injury to the right side as a result of a road accident in Pakistan. He required vigorous resuscitation and a laparotomy revealed lacerations to the right lobe of the liver which were sutured. He made a slow post-operative recovery but was admitted to hospital on return to England 6 weeks later complaining of anorexia and weight loss. He was thin and wasted, afebrile, with dullness at the right lung base and fullness in the right upper quadrant of the abdomen, with signs of infection in the abdominal incision. The liver function tests were abnormal with elevation of the alkaline phosphatase to $965 \mathrm{IU} / 1$ (normal $<130 \mathrm{IU} / 1$ ) and the gammaglutamyl transpeptidase to $309 \mathrm{IU} / 1$ (normal $<45 \mathrm{IU} / 1)$ although the bilirubin was normal. The serum albumin was low at $30 \mathrm{~g} / 1$ (normal $35-50 \mathrm{~g} / \mathrm{l}$ ) and the serum sodium low at $128 \mathrm{mmol} / \mathrm{l}$. An ultrasound scan of the abdomen showed a large subcapsular collection of fluid adjacent to the liver and this was confirmed by computerized tomography (Figure 1). Ultrasound guided aspiration revealed clear bile which was culture negative. A pigtail catheter left in situ continued to drain $(2250 \mathrm{ml}$ in the first 48 hours)

Correspondence: J.R. Crampton M.A., M.R.C.P.

Accepted: 4 September 1986 and there was persistent biliary leakage over the first 10 days. Introduction of contrast down the catheter failed to indicate the site of any fistula so that an ERCP was performed, since it was feared that spontaneous closure might not occur. The ERCP (Figures $2,3)$ clearly demonstrated passage of contrast from a branch of the right hepatic duct into a cystic cavity although the remainder of the biliary system was normal. Because of his poor nutritional status it was felt that operative intervention should be deferred and he underwent a period of parenteral nutrition. Over the next month the drainage volume decreased considerably to less than $200 \mathrm{ml}$ per 24 hours and a sinogram indicated that the cavity had shrunk. The residual cavity became secondarily infected and the draining catheter blocked so that a spontaneous sinus developed to the skin in the midline. This was therefore laid open to allow drainage and healing to occur. No further bile drained from the wound indicating that the fistula had closed. Four months

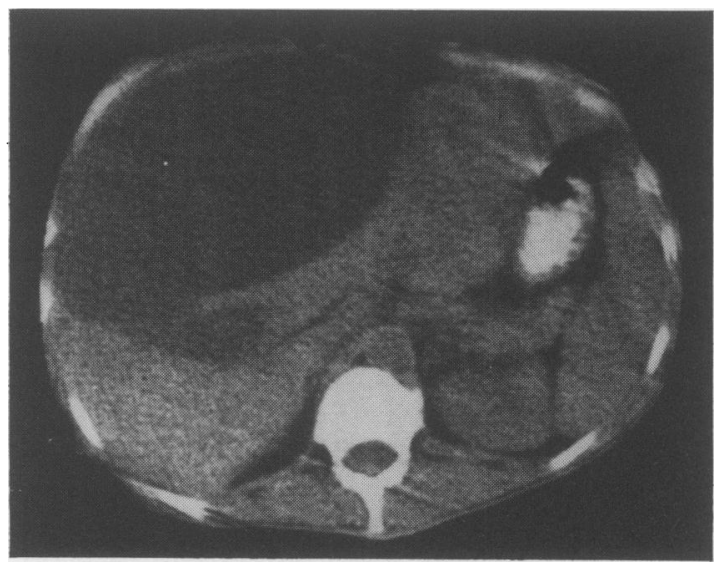

Figure 1 CT scan showing a huge subcapsular fluid collection displacing the liver.

(C) The Fellowship of Postgraduate Medicine, 1987 


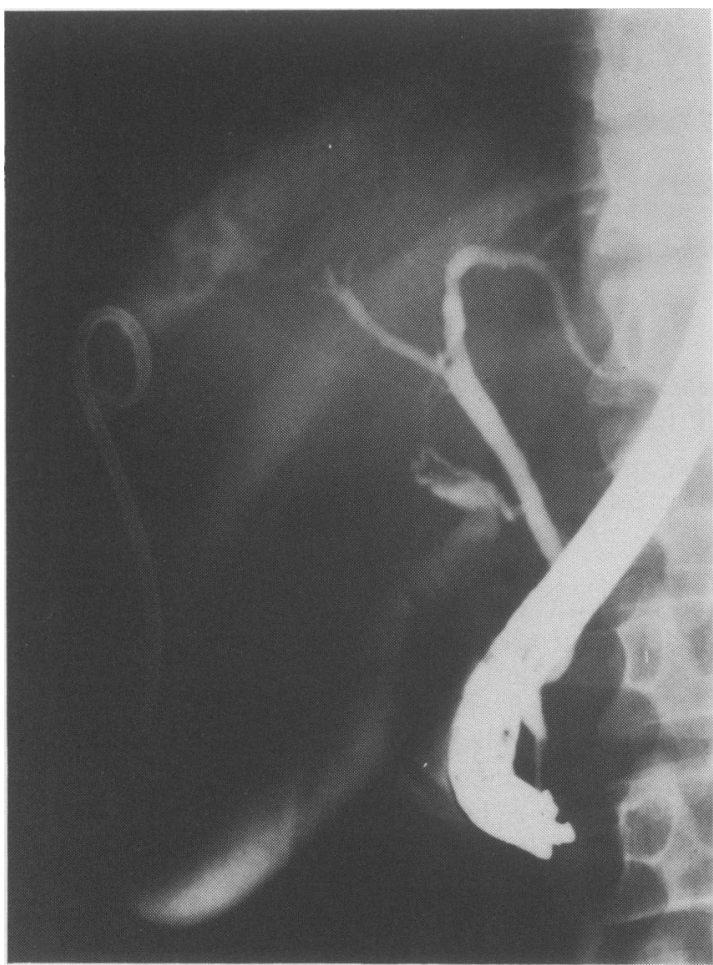

Figure 2 ERCP demonstrating normal common bile duct and gall bladder but showing leakage of contrast to an ill defined space in which the pigtail catheter is seen to lie.

later the wound had healed completely and he had gained his former weight and was well.

\section{Discussion}

Hepatic trauma is becoming a commoner surgical emergency owing to the greater volume and speed of road traffic and the increasing incidence of civil violence. Nonetheless, the British experience of this problem is relatively trivial compared with that in the USA. Large series there, however, indicate that biliary fistula is a relatively rare sequela occurring in between $0.5 \%^{1}$ and $2.6 \%{ }^{2}$ of all cases of hepatic trauma even where gunshot, stab wounds and other penetrating trauma form the majority of cases. Most cases present early and failure of spontaneous closure is very rare. From the combined total of 2311 cases of hepatic trauma in the two cited American series posttraumatic biliary fistula occurred in $\mathbf{4 6}$ but all closed with conservative management. Persistent drainage over a long period of time however does rarely occur. Fistulation is more likely to be persistent in the

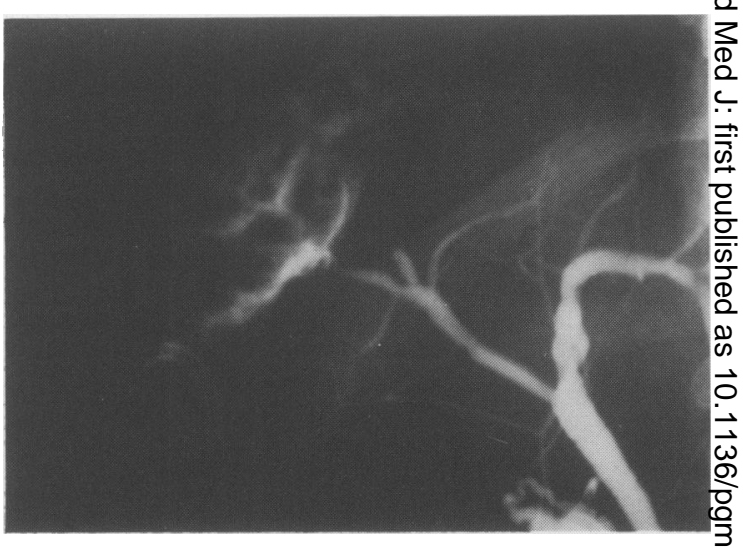

Figure 3 After further injection of contrast it is clear that a branch of the right hepatic duct is draining into the cavity.

presence of distal biliary obstruction, extensive loss of bile duct wall or infection. Smith et al. ${ }^{3}$ have described ${ }^{\circ}$ three cases of persistent fistula who continued to drain large volumes over many months and have discussed $\Phi$ the available surgical options: simple suture, fistulo-을 jejunostomy and fistulocholecystostomy.

When persistent biliary drainage from a fistuda occurs the question of determining the site of le ${ }^{3}{ }_{\infty}$ arises. Possible imaging techniques include percuta ${ }_{-1}^{-}-v$ eous, intravenous, or endoscopic cholangiograph, biliary scintigraphy and ultrasound. Contrast can also be introduced into the cutaneous orifice and a sin- $\frac{-}{0}$ ogram obtained. In our patient the sinogram failed to opacify any elements of the biliary tree even in the $\frac{}{\circ}$ presence of large volumes of bile drainage. Although $\overrightarrow{\vec{F}}$ scintigraphy is capable of confirming biliary leakage $\frac{0}{3}$ the precise anatomical location may be difficult to determine owing to the low anatomical resolution of the scan. Percutaneous cholangiography is invasive and may fail in the absence of dilated ducts. Intraven- 3 ous cholangiography is insufficiently accurate and the incidence of reaction to the contrast is high. ERCP in 3 . this context has been advocated only once in a letter ${ }^{4} \delta$ but in a case presenting early after gunshot wound. Our case is unusual in that the late presentationo following blunt trauma raised the possibility of persistence of the fistula so that the anatomical delineation of the site was more pressing. We believe that ERCP has many advantages as an imaging technique in these. circumstances. The whole biliary system can be N examined and the precise site of the fistula determined. N In addition distal biliary obstruction can be excluded $\sigma$ and if present may be treatable endoscopically using the techniques of endoscopic sphincterotomy and stone extraction. Endoscopic stenting has been used $\stackrel{\oplus}{?}$ for post-operative biliary fistulae ${ }^{5}$ and may also have $a_{0}$ role in the post traumatic variety. These considerable ${ }_{0}^{-}$ 
advantages lead us to recommend the use of ERCP in the diagnosis and management of patients with persistent post-traumatic biliary fistula.

\section{References}

1. Trunkey, D.D., Shires, G.T. \& McClelland, R. Management of liver trauma in 811 consecutive patients. Ann Surg 1974, 179: 722-728.

2. Defore, W.W., Mattox, K.L., Jordan, G.L. \& Beall, A.C. Management of 1590 consecutive cases of liver trauma. Arch Surg 1976, 111: 493-497.

3. Smith, E.E.J., Bowley, N., Allison, D.J. \& Blumgart, L.H.

\section{Acknowledgements}

The authors wish to thank Professor M. Irving for permission to report this case.

The management of post traumatic intrahepatic cutaneous biliary fistulas. Brit $J$ Surg 1982, 69: 317-318 .

4. Nelson, A.M. Demonstration of a traumatic biliary fistula by ERCP. Gastrointest Endosc 1984, 30 (5): 315 (letter).

5. Smith, A.C., Schapiro R.H., Kelsey P.B. \& Warshaw A.L. Successful treatment of non-healing biliary cutaneous fistulas with biliary stents. Gastroenterology 1986, 90: $764-769$ 